\title{
A expressiva importância da humanização no trabalho de parto
}

\author{
The expressive importance of humanization in labor \\ La importancia expresiva de la humanización en el trabajo
}

Gisele de Jesus Batista

ORCID: https://orcid.org/0000-0001-9832-3307

Universidade de Gurupi, Brasil

E-mail: gysabatysta@gmail.com

Carla Thailenna Jorge Pereira

ORCID: https://orcid.org/0000-0001-6278-7564

Universidade de Gurupi, Brasil

E-mail: thailenna1991@gmail.com

Fernanda Roques Felipe

ORCID: https://orcid.org/0000-0002-1661-6876

Universidade de Gurupi, Brasil

E-mail: nandaroquesf@gmail.com

Kassio Maluar Gonsalves Luz

ORCID: https://orcid.org/0000-0002-4574-0251

Universidade Federal do Tocantins, Brasil

E-mail: kassiomaluar@gmail.com

Milena Matos da Cruz

ORCID: https://orcid.org/0000-0002-9875-8906

Instituto Presidente Antonio Carlos, Brasil E-mail:milenamc_5@gmail.com

Deyla Jordana de Oliveira Silva

ORCID: https://orcid.org/0000-0001-7543-2690

Instituto Presidente Antonio Carlos, Brasil

E-mail: deylajordana@hotmail.com

Jonathan Jean Vilhaba

ORCID: https://orcid.org/0000-0002-6018-1195 Universidade de Gurupi, Brasil

E-mail: vilhaba@gmail.com

Natália Rose de Almeida Leite Furtado

ORCID: https://orcid.org/0000-0002-6907-0037

Instituto Presidente Antonio Carlos, Brasil E-mail: nataliaralf94@gmail.com

Daniela de Souza silva

ORCID: https://orcid.org/0000-0001-5558-5980 Universidade de Gurupi, Brasil

E-mail: danielassilva@unirg.edu.br

Juciely Magalhaes da Silva

ORCID: https://orcid.org/0000-0002-0845-9102 Universidade de Gurupi, Brasil E-mail: jucielyms@unirg.edu.br

Andrênia Soares Montes

ORCID: https://orcid.org/0000-0001-9806-7155 Universidade de Gurupi, Brasil

E-mail: andreniasm@hotmail.com

\begin{abstract}
Resumo
Introdução: $\mathrm{O}$ termo humanizar nos remete a uma assistência que valorize a qualidade do cuidado do ponto de vista técnico, associado ao respeito dos direitos do paciente, de sua subjetividade e referências culturais, como também a valorização do profissional e do diálogo intra e interequipes.O momento do parto, envolve muitas questões biológicas, sociais e psicológicas para a mulher e seu bebe, o medico precisa entender a sensibilidade que envolve a gestante nesse momento. Metodologia:Trata-se de uma revisão narrativa da literatura Os dados utilizados nesta pesquisa caracterizam-se como secundários, ou seja, fundamentados em dados já tratados, retirados de livros e obras para mostrar o conceito, tratamento e suas particularidades.Resultados: A necessidade de profissionais de saúde capacitados e sensibilizados na assistência ao pré-natal e no período do parto é de fundamental importância, visto que esse é um momento doloroso e desconfortável para a mulher. Portanto, torná-lo menos traumático deve ser um dos objetivos de toda equipe assistencial.Conclusão: Após a leitura e análise dos artigos, verificou-se que para a
\end{abstract}


enfermagem possa contribuir na melhoria da qualidade da assistência á saúde da mulher, é fundamental que as intervenções realizadas possam garantir a saúde física e psíquica, o respeito e a dignidade, oferecendo assistência humanizada durante todo o ciclo gravídico puerperal, entendendo a gestação e o parto como eventos fisiológicos, desenvolvendo o protagonismo do parto à mulher, onde participa ativamente das decisões podendo expressar seus desejos e escolhas, decidindo junto com a equipe o melhor método a ser adotado.

Palavras-chave: Medicina obstétrica; Parto humanizado; Humanização da assistência.

\begin{abstract}
Introduction: The term humanize refers us to care that values the quality of care from a technical point of view, associated with respect for the patient's rights, their subjectivity and cultural references, as well as the valuation of the professional and of intra- and inter-team dialogue. The moment of delivery, involves many biological, social and psychological issues for the woman and her baby, the doctor needs to understand the sensitivity that surrounds the pregnant woman at that time. Methodology: It is a narrative review of the literature The data used in this research are characterized as secondary, that is, based on data already treated, taken from books and works to show the concept, treatment and its particularities.Results: The need of trained and sensitized health professionals in prenatal care and in the delivery period is of fundamental importance, since this is a painful and uncomfortable moment for women. Therefore, making it less traumatic should be one of the goals of the entire care team. Conclusion: After reading and analyzing the articles, it was found that for nursing to contribute to improving the quality of women's health care, it is essential that the interventions carried out can guarantee physical and mental health, respect and dignity, offering humanized care throughout the pregnancy and childbirth cycle, understanding pregnancy and childbirth as physiological events, developing the role of the woman in childbirth, where she actively participates in decisions being able to express their wishes and choices, deciding together with the team the best method to be adopted.
\end{abstract}

Keywords: Obstetric medicine; Humanized birth; Humanization of assistance.

\begin{abstract}
Resumen
Introducción: El término humanizar nos refiere a una asistencia que valora la calidad de la atención desde un punto de vista técnico, asociado al respeto de los derechos del paciente, su subjetividad y referentes culturales, así como la valorización del profesional y el Diálogo intra e interequipo El momento del parto, involucra muchas cuestiones biológicas, sociales y psicológicas para la mujer y su bebé, el médico necesita comprender la sensibilidad que envuelve a la gestante en ese momento. Metodología: Es una revisión narrativa de la literatura Los datos utilizados en esta investigación se caracterizan como secundarios, es decir, basados en datos ya tratados, extraídos de libros y obras para mostrar el concepto, tratamiento y sus particularidades. Resultados: La necesidad de Profesionales de la salud capacitados y sensibilizados en la atención prenatal y en el parto es de fundamental importancia, ya que este es un momento doloroso e incómodo para la mujer. Por lo tanto, hacerla menos traumática debe ser una de las metas de todo el equipo de atención.Conclusión: Luego de leer y analizar los artículos, se encontró que para que la enfermería contribuya a mejorar la calidad de la atención de salud de la mujer, es fundamental que puede garantizar la salud física y mental, el respeto y la dignidad, ofreciendo cuidados humanizados durante todo el ciclo del embarazo y parto, entendiendo el embarazo y el parto como eventos fisiológicos, desarrollando el rol de la mujer en el parto, donde participa activamente en las decisiones pudiendo expresarse sus deseos y elecciones, decidiendo junto con el equipo el mejor método a adoptar.
\end{abstract}

Palabras clave: Medicina obstétrica; Nacimiento humanizado; Humanización de la asistencia.

\title{
1. Introdução
}

No passado, o partejar era realizado por curandeiras, parteiras ou comadres, que conheciam o processo do parto e puerpério de acordo com suas experiências próprias, tratando-o humanitariamente. O atendimento ao nascimento, na época, era uma atividade desvalorizada pelo profissional médico, sendo deixado aos cuidados femininos (Sena et al., 2012).

O parto representa uma transição importante na vida da mulher e da família. É considerado um processo psicossomático, no qual o comportamento da gestante ou parturientevai depender, além da própria evolução do trabalho de parto, de seu nível de informação, de sua história pessoal, contexto socioeconômico, personalidade e simbolismo. Portanto, constitui um momento em que ela necessita de apoio e compreensão para poder enfrentá-lo o mais naturalmente possível, sabendo que pode e deve participar ativamente, obtendo assim, conforto físico e psíquico (Tomeleri et al., 2007)

O apoio emocional de um acompanhante de escolha da parturiente é eficaz para que a mulher possa suportar a dor e tensão. Neste sentido, o acompanhante necessita do apoio e colaboração dos profissionais de saúde na condução adequada da assistência à mulher. É importante que os profissionais de saúde estejam sensibilizados quanto à relevância da presença 
do acompanhante para parturiente no decorrer do trabalho de parto, como também precisam estar preparados para executarem suas atividades junto ao acompanhante e parturiente, informando-os sobre a evolução e condutas a serem realizadas durante o processo de nascimento. São atitudes simples, mas eficazes que podem influenciar positivamente a realidade da assistência da mãe e seu concepto (Moura et al., 2007).

O termo humanizar nos remete a uma assistência que valorize a qualidade do cuidado do ponto de vista técnico, associado ao respeito dos direitos do paciente, de sua subjetividade e referências culturais, como também a valorização do profissional e do diálogo intra e interequipes. A humanização da assistência traduz a necessidade de mudanças na compreensão do parto, como experiência humana e, para quem o assiste, uma transformação "no que fazer e que horas fazer", diante do sofrimento do outro (Souza et al., 2011)

Com isso, a humanização do parto representa uma nova maneira de assistir a mulher, a criança e a família é estar sempre ao lado, prestando-lhes o suporte necessário de forma individualizada, garantindo, assim uma melhor integralidade da assistência para mãe/bebê. É saber identificar os riscos antes que eles ocorram para que se possa garantir ao ser humano o direito à "vida". (Silva, 2007)

A humanização é de extrema importância nesse período único e muito intimo na vida de quem gesta.As mulheres precisam receber uma assistente livre de preconceitos e traumas que marcarão suas vidas e de seus filhos.O parto preciso acontecer de modo mais natural e tranqüilo possível, e a medicina tem papel fundamental nesse processo, evitando condutas e intervenções desnecessárias.

\section{Metodologia}

Tratou-se de uma revisão narrativa da literatura, uma vez que é apropriada para descrever, discutir e analisar de forma ampla a literatura publicada sobre determinado tema, sob o ponto de vista teórico ou contextual (Winck; Brüggemann, 2010).

A revisão bibliográfica foi realizada através de pesquisas em artigos científicos contidos na Biblioteca Virtual em Saúde a partir dos seguintes descritores: medicina Obstétrica, Parto Humanizado e Humanização da Assistência. Além desses, foram consultados sites de órgãos oficiais de reconhecida relevância para o assunto onde foram encontradas uma publicação do Ministério da Saúde (Caderno de Atenção da Rede Cegonha) e duas monografias de Enfermagem que tratavam sobre o assunto pesquisado.

Inicialmente foi realizada a leitura dos resumos das publicações localizadas a fim de identificar quais atendiam aos seguintes critérios de inclusão: Discorrer sobre a atuação do medico no parto humanizado, serem publicados em português, entre os anos de 2005 e 2013 e estarem disponíveis gratuitamente nas fontes pesquisadas para leitura naíntegra.

Após a busca e seleção do material, este foi submetido à leitura e organizado emforma de tópicos de acordo com as diferentes temáticas encontradas. Nesses tópicos, as ideias entre os diversos autores foram explicitadas e relacionadas de modo a estabelecer uma análisedescritiva e reflexiva acerca da temática.

Foram respeitados os princípios éticos da pesquisa ao citar as obras e assim resguardar os direitos autorais dos estudos citados.

\section{Resultados e Discussão}

O parto sofreu ao longo dos anos muitas modificações com relação ao seu modeloassistencial. Até o século XVI o parto era considerado "assunto de mulher" ao qual existiamas parteiras que assistiam as mulheres durante o período de parturição e que criavam um climaemocional favorável para a parturiente (Silva, 2007). 
Castro e Clapis (2005) afirmam que com o passar dos anos, o ato fisiológico de parir e nascer passou a ser visto como patológico, privilegiando a técnica medicalizada e despersonalizada, em detrimento do estímulo, apoio e carinho à mulher que vivencia essa experiência. Ainda segundo os autores no processo de humanização há necessidade de diminuição do índice de cesarianas e da melhoria da assistência à mulher, apontando tambémo excesso de atitudes intervencionistas que levam à despersonalização do seu papel no parto. Dessa forma, entendem que a assistência precisa ser desmedicalizada para que se consiga alcançar a humanização.

A respeito da perspectiva de humanização da assistência à mulher no parto, Silva (2011) destaca:

O conceito de atenção humanizada é amplo e envolve um conjunto de conhecimentos, práticas e atitudes que visam à promoção do parto e do nascimento saudáveis e a prevenção da morbimortalidade materna e perinatal. Tem início no pré-natal estendendo-se até o puerpério, procurando garantir que a equipe de saúde realize procedimentos comprovadamente benéficos para a mulher e o bebê, evitando intervenções desnecessárias e preservando sua privacidade e autonomia. Silva (2011) ainda diz que, nesse sentido, a assistência humanizada proporciona às mulheres um forte sentimento de confiança e segurança durante o parto e ao cuidar de seu filho. Muitas têm uma experiência maravilhosa de autotransformação, sentindo-se capazes em seu novo papel social. Esta experiência estimula a conscientização e o interesse pela sociedade, tendo como consequência o fortalecimento social.

O conceito de humanização do parto pode ser bastante diversificado, porém, háum movimento defendendo-o como um processo que respeita a individualidade das mulheres, valorizando-a como protagonista e permitindo a adequação da assistência à cultura, crenças, valores e diversidade de opiniões dessas pessoas. Assim, "humanizar o parto é respeitar e criar condições para que todas as dimensões do ser humano sejam atendidas: espirituais, psicológicas e biológicas" (Castro; Clapis, 2005).

Para Silva (2007), a humanização abrange aspectos fundamentais, o qual dizrespeito à convicção de que é dever das unidades de saúde receber com dignidade a mulher, seus familiares e recém-nascidos, ao qual requer uma atitude ética e solidária por parte dos profissionais de saúde, organizar a instituição de modo a criar um ambiente acolhedor,adotando condutas hospitalares que rompam com o tradicional isolamento imposto a mulher.

A humanização privilegia o bem-estar da mulher e do bebê ao considerar os processos físiológicos, psicológicos e o contexto sociocultural, caracterizado pelo acompanhamento contínuo de gestação e parturição. Nessa concepção, o parto é favorecido em centros de parto normal, casas de parto e hospitais. A presença de acompanhantes é incentivada e a mulher tem maior liberdade para escolher o modo e a posição que lhe é mais confortável para ter seu filho. (Brasil, 2013, p.11)

Dessa forma, o acompanhante da gestante precisa ser visto como um coringa no processo de acompanhamento e boa evolução no trabalho de parto, sendo um grande beneficio para gestante e para os profissionais que passam a ter um papel de suporte na orientação e acompanhamento do trabalho de parto. É importante que lembrarmos que o acompanhamento não e um funcionário da instituição e sim uma pessoa que também participa do processo do cuidado, e deve ser acolhido também, (Santos et al., 2012)

Essa relação da participação da família pode ser vista no estudo de Longo et al (2010), no qual afirma que o acompanhante escolhido traz consigo significados diversos quanto à sua participação podendo refletir positivamente no comportamento da parturiente durante a parturição. Nesse sentido, o acompanhante é parte do imaginário da gestante quandoesta pensa no momento do parto. Nele é depositada pela parturiente a segurança de ter alguémpróximo e confiável. Os autores relatam ainda que o cotidiano tem mostrado que a participação do acompanhante durante o processo de parturição não envolve outra questão senão o apoio emocional, que a equipe de saúde nem sempre tem condições e tempo 
para oferecer à parturiente. Por outro lado, as demais necessidades assistenciais são de responsabilidade exclusiva da equipe de saúde. Nessa perspectiva compartilhar este momento do parto e nascimento, contando com a parceria do acompanhante, pode ser um facilitador do trabalho para a parturiente.

É válido ressaltar que a humanização da assistência ao parto exige, principalmente, que a atuação do profissional respeite os aspectos de sua fisiologia, não intervenha de forma desnecessária, reconheça os aspectos sociais e culturais do parto e pós-parto, e ofereça o suporte emocional à mulher e à sua família. O que facilitará a formação dos laços afetivos familiares e o vínculo mãe-bebê (Fialho, 2008).

Diante disso, não podemos realizar intervenções que causem desconforto a gestante e o bebe, visto que os traumas psicologicos podem marcar a vida da mulher para sempre.

\subsection{Programa de humanização no pré-natal e Nascimento do Ministério da Saúde noBrasil}

Durante muitos anos, o governo brasileiro não apresentava posição explícita frente à questão da saúde da mulher. A partir dos anos 80, pressionado pelos profissionais de saúde, movimentos de mulheres e outras instituições da sociedade civil organizada, iniciaram-se algumas mudanças relacionadas à forma de atendimento à mulher, que valorizavam a maior participação, informação e consciência dos seus direitos, favorecendo o empoderamento e cidadania (Cardoso et al, 2007)

A parturição mais humanizada deve ser um objetivo principal para todas as maternidades nacionais, com essa finalidade foi criado o programa do Ministério da saúde, que em junho de 2000, criou o Programa de Humanização no PréNatal e Nascimento (PHPN). O Programa tem como objetivo integrar a atenção obstétrica integrada, qualificando e humanizando de forma articulada dos estados, municípios e das unidades de saúde nestas ações (Santos et al., 2012)

Nesta perspectiva, o Ministério da Saúde, vem ao longo das últimas décadas propondo políticas de atenção integral à saúde da mulher e da criança, que assumem compromissos com a garantia dos direitos de cidadania, sexuais e reprodutivos. O Programade Humanização no Pré-Natal e Nascimento (PHPN) têm como obejeto principal a estratégia de aumentar a segurança e cobertura da qualidade da assistência ao pré-natal, durante o parto e puerperio das gestantes e do seu binomio, dando direito a cidadania e vida. (Souza et al., 2011)

O PHPN estabeleceu ainda uma tática para assistenciar a maioria dos municípios a programar essas ações, introduzindo novos recursos para o custeio dessa assistência e transferindo-os diante do cumprimento de critérios mínimos, necessários para melhorar aqualidade da assistência. As exigências foram:

Realizar a primeira consulta de pré-natal até o mês quatro de gestação; assegurar a realização dos procedimentos: no mínimo, seis consultas de pré-natal, sendo, preferencialmente, uma no primeiro trimestre, duas no segundo e três no terceiro trimestre da gestação; uma consulta no puerpério, até 42 dias após o nascimento; exames laboratoriais: (a) $\mathrm{ABO}-\mathrm{Rh}$, na primeira consulta; (b) VDRL, um exame na primeira consulta e outro próximo à trigésima semana da gestação; (c) Urina rotina, um exame na primeira consulta e outro próximo à trigésima semana da gestação; (d) Glicemia de jejum, um exame na primeira consulta e outro próximo à trigésima semana da gestação; (e) Hemoglobina/Hematócrito na primeira consulta; oferta de testagem anti-HIV, com um exame na primeira consulta, naqueles municípios com população acima de cinquenta mil habitantes; aplicação de vacina antitetânica até a dose imunizante (segunda) do esquema recomendado ou dose de reforço em mulheres já imunizadas. (Souza, 2007)

Pereira e Bento (2011) afirma que na contemporaneidade, as políticas de saúde damulher vêm ampliando o cuidado à mulher sob a perspectiva de gênero, este compreendido como elemento constitutivo das relações sociais entre homens e mulheres é resultante de uma construção social e histórica, que determina os modelos culturais e comportamentais de masculinidade e feminilidade, representando uma forma primeira de significação de poder. 
Contudo, humanizar e qualificar a atenção em saúde é aprender a compartilhar saberes e reconhecer direitos. Portanto, procurar articular vários campos de conhecimento necessário no cuidado integral à mulher e ser um instrumento de garantia dos direitos das mulheres nas ações de saúde (Pereira; Bento, 2011)

\subsection{Centros de Parto Normal}

A discussão acerca da segurança da assistência a parturientes em modalidades nãohospitalares veio à tona no Brasil com a edição da Portaria nº 985/1999 do Ministério da Saúde, que autoriza e regulamenta os Centros de Parto Normal (CPN) no âmbito do Sistema Único de Saúde. Os CPN constituem uma estratégia para reduzir a mortalidade materna e perinatal, e, oferecendo uma assistência humanizada e de baixo intervencionismo, devem contribuir para uma melhoria da assistência ao parto normal. Ainda de acordo com o autor, desde o primeiro CPN, fundado em 1975 pela Maternity Center Association em Nova York, Estados Unidos, observa-se um crescimento constante dessa modalidade assistencial no mundo inteiro. Difundiuse o discurso acerca das vantagens de um local de parto, fora do hospital, acolhedor e similar ao domicílio, porém com equipamentos e infraestrutura técnica e recursos humanos adequados para as mulheres com gestação de baixo risco (Campos; Lana, 2007).

Ainda de acordo com Campos e Lana (2007) o parto de uma gestação de baixo risco pode ser assistido com segurança no domicílio, numa casa de parto ou na maternidade de um hospital, sendo a enfermeira-parteira o profissional mais adequado para essa função. Define a OMS o parto de baixo risco como aquele que tem início espontâneo entre 37 e 42 semanas completas, sem nenhum fator de risco identificado, mantendo-se esse quadro durantetodo processo, que culmina com o nascimento de um recém-nascido em posição cefálica. Ainda para a OMS, o parto normal é um processo natural, fisiológico e, consequentemente, não deve sofrer intercorrências no seu curso.

$\mathrm{O}$ CPN destina-se à assistência ao parto normal sem distocia, inserido no sistema de saúde local, que funciona de maneira intra ou extra-hospitalar. Quando localizado fora do ambiente hospitalar é conhecido como casa de parto e tem o hospital como referência para remoções em um período máximo de uma hora. Os resultados da assistência em CPN têm apontado satisfação da mulher e sua família, além da redução de intervenções desnecessárias. (Schneck et al, 2012)

No Centro de Parto Normal, a parturiente e seu acompanhante são informados, constantemente, pela enfermeira obstétrica que os assiste, sobre a evolução do trabalho de parto e sobre eventuais mudanças de conduta para que possam colaborar durante todo o processo do trabalho de parto ativo até o nascimento. À cliente em trabalho de parto é permitido expressar seus sentimentos quanto aos procedimentos com os quais não concorda, cabendo à equipe, dentro de suas possibilidades, mudar a conduta de modo que a parturiente sinta-se segura em relação à assistência prestada. (Machado; Praça, 2006)

Na Casa de Parto são realizadas práticas educativas como orientações, oficinas, dinâmicas, jogos, dramatizações, entre outras. Estas práticas abordam questões relacionadas à gestação, ao trabalho de parto, à amamentação ao vínculo, aos direitos da gestante, ao gêneroe sexualidade, às tecnologias de cuidados de enfermagem, aos cuidados com o recém-nascido e no pósparto, promotoras das ações de cuidado da enfermeira e medica obstétrica (Pereira; Bento, 2011).

Estes espaços são ocupados pelo cuidado profissional de enfermeiras obstétricas e visam oferecer condições favoráveis que apoiem e facilitem o processo não invasivo no parto.Neste sentido, as Casas de Parto, além de serem mais uma opção para que a mulher possa escolher sua maneira de parir, constituem-se também numa poderosa estratégia política dos defensores do modelo humanizado e desmedicalizado de assistência ao parto e nascimento (Progianti; Costa, 2008).

Os Centros de Parto Normal nasceram com o objetivo de ofertar uma ambiente familiar de dignidade e semelhante ao ambiente que a gestante vive, garantindo a segurança à mãe e seu filho, oferecendo- lhes recursos modernos adequados em 
casos de eventual necessidade. Seguem um padrão de procedimentos previamente estabelecidos e que direcionam as ações que realizam (Machado; Praça, 2006)

Diante disso, as Casas de Parto representam um local onde as enfermeiras assistem as mulheres com qualidade e de forma humanizada, atuando com competência, demonstrando o saber-fazer técnico e a tomada de decisão para a garantia do bem estar da mãe e de seu filho. O cuidado não é apenas a técnica, os procedimentos assistenciais. O cuidar envolve a preocupação, o interesse e a motivação, assim como a gentileza, o respeito e a consideração pelo outro, o ser do cuidado. Por outro lado, há a intencionalidade de promover o bem-estar, de manter o ser seguro e confortável, de oferecer apoio, de minimizar riscos e reduzir a sua vulnerabilidade. (Pereira; Bento, 2011)

\section{Conclusão}

A necessidade de profissionais de saúde capacitados e sensibilizados na assistência ao pré-natal e no período do parto é de fundamental importância, visto que esse é um momento doloroso e desconfortável para a mulher. Portanto, torná-lo menos traumático deve ser um dos objetivos de toda equipe assistencial. Isso pode ser feito de forma simples e com práticas que nem sempre envolvem a medicalização do parto, ou seja, práticas demassagem, deambulação e acolhimento da equipe multiprofissional que facilitam o parto e favorecem uma aproximação profissional à paciente. Dessa forma, a atuação o profissional medico obstetra no processo de trabalho de parto, quando realizada de maneira adequada e humanizada, pode proporcionar a parturiente um momento de satisfação e bem-estar. Aequipe precisa esta preparada para acolher as gestantes e seus familiares, respeitando os diversos aspectos individuais, culturais, emocionais envolvidos no processo. Para isso a atuação do medico obstetra requer uma visão humanística, ou seja, a necessidade de compreender o outro.

A autonomia da mulher no momento do parto também deve ser valorizada, uma vez que ela e o bebê são os protagonistas desse momento tão importante na vida dos dois, um está chegando à vida e o outro está proporcionando tal acontecimento. A mulher precisa ser acolhida por toda equipe e ela precisa perceber isso, ou seja, a equipe precisa demonstrar alémdo profissionalismo o carinho pela parturiente. O profissionalismo inclui boa assistênciatécnica, informação e orientação sobre seus direitos neste período e também no puerpério. O enfermeiro frente à assistência ao parto assume um papel de extrema importância, já que ele possui um contato direto com a mulher, e a capacidade de identificar e compreender seu sofrimento, suas necessidades individuais, prestando uma assistência humanizada e de qualidade.

\section{Referências}

Brasil. Ministério Da Saúde. Gravidez, parto e nascimento com saúde, qualidadede vida e bem-estar. 11p.

Castro, J. C., \& Clapis, M. J. (2005). Parto humanizado na percepção das enfermeiras obstétricas envolvidas com a assistência ao parto. Rev Latino-am Enfermagem. 13(6): 960-7, novembro-dezembro.

Cardoso, A. M. R., Santos, S. M. \& Mendes, V. B. (2007). O pré-natal e saúde da mulher na gestação: um processo educativo? Diálogos Possíveis, Brasília, janeiro/junho.

Campos, S. E. V., \& Lana, F. C. Resultados da assistência ao parto no centro de parto normal dr. david capistrano da costa filho em belo horizonte, minas gerais, brasil. Cad. SaúdePública, 23(6):1349-1359

Fialho, T. C. O papel do enfermeiro no parto humanizado. 2008. 38p. Trabalho de Conclusão de Curso (Especialização em Saúde Pública) Educação Avançada Ltda.,

Gomes, M. L., Moura, M. A. V., \& Souza, I. E. A. A prática obstétrica da enfermeira no parto institucionalizado: uma possibilidade de conhecimento emancipatório. Texto Contexto Enferm, 22(3): 763-71,

Longo, C. S., Andraus, L. M., \& Barbosa, M. A. Participação do acompanhante na humanização do parto e sua relação com a equipe de saúde. Rev. Eletr. Enf, 12(2): 386-91

Machado, N. X., \& Praça, N. S. Centro de parto normal e assistência obstétrica centrada nasnecessidades da parturiente. Rev Esc Enferm, 40(2): 274-9 
Research, Society and Development, v. 10, n. 7, e53510716656, 2021

(CC BY 4.0) | ISSN 2525-3409 | DOI: http://dx.doi.org/10.33448/rsd-v10i7.16656

Moura, F. M. J. S. P., Crizostomo, C. D., Nery, I. S., Mendonça, R. C. M., Araujo, O. D., \& Rocha, S, S. A humanização e a assistência de enfermagem ao parto normal. Rev BrasEnferm, 60(4):452-5

Nunes, A. R., Silva, L. O., \& Silva, E. A. M. Contribuições do profissional da enfermagem noparto humanizado. Seminário de Pesquisas,

Pereira, A. L. F., \& Bento, A. D. Autonomia no parto normal na perspectiva das mulheres atendidas na casa de parto. Rev Rene, 12(3): 471-7

Progianti, J. M., Costa, R. F. A negociação do cuidado de enfermagem obstétrica através das práticas educativas na casa de parto. Esc Anna Nery Rev Enferm, 12 (4): 789-92

Santos, R. B., \& Ramos, K. S. Sistematização da assistência de enfermagem em centro obstétrico. Rev Bras Enferm, 65(1): 13-8

Santos, G. S., Souza, J. L. O., Almeida, L. S., \& Gusmao, M. H. A importância do enfermeiro no atendimento humanizado no pré-parto. Diálogos Ciência, 224-228

Santos, I. S., \& Okazaki, E. L. F. J. Assistência de enfermagem ao parto humanizado. Rev Enferm, 13(1): 64-8, 2012.

Schneck, C. A., Riesco, M. L. G., Bonadioll, I. C., Diniz, C. S. G., Junqueira, S.M., \& Oliveira, V. Resultados maternos e neonatais em centro de parto normal peri-hospitalar e hospital. Rev Saúde Pública, 46(1):77-86,

Sena, C. D., Santos, T. C. S Carvalho, C. M. F., Sá, A. C., \& Paixao, G. P. N. Avanços e retrocessos da enfermagem obstétrica no brasil. Rev Enferm, 2(3): 523-529

Silva, L. R., Christoffel, M. M., \& Souza, K, V. história, conquistas e perspectivas no cuidado à mulher e à criança. Texto Contexto Enferm, 14(4): 585-93

Silva, P. A. Humanização no cotidiano do enfermeiro (a) ao cuidar de mulheres durante o parto. 2007. 44f. Trabalho de Conclusão de Curso (Graduação em Enfermagem)- Faculdade de Ciências da Saúde, Centro Universitário de Brasília

Souza, G. T., Gaiva, M. A., \& Modes, A. S. S. P. A humanização do nascimento: percepção dos profissionais de saúde que atuam na atenção ao parto. Rev Gaúcha Enferm, 32(3): 479-86

Tomeleri, K. R., Pieri, F. M., Violin, M. R., Serafim, D., \& Marcon, S. S. "Eu vi meu filho nascer": vivência dos pais na sala de parto. Rev Gaúcha Enferm, 28(4): 497-504

Vargens, O. M. C., Progianti, J. M., \& Silveira, A. C. F. O significado de desmedicalização da assistência ao parto no hospital: análise da concepção de enfermeiras obstétricas. Rev Esc Enferm , 42(2):339-46,

Winck, D. R., \& Bruggemannl, O. M. Responsabilidade legal do enfermeiro emobstetrícia. Rev Bras Enferm, 63(3): 464-9 